\title{
An Outbreak of Epidemic Keratoconjunctivitis Due to Adenovirus Type 8 in a Babies Home
}

\author{
Shunzo Chiba, Masao Umetsu, Tatsuru Yamanaka, \\ Shoko Hort, Tooru Nakao and Sekt Fukur* \\ Department of Pediatrics, Sapporo Medical College, Sapporo \\ and Hokkaido Central Babies Home,* Sapporo
}

Chiba, S., Umetst, M., Yamanaka, T., Hort, S., Nakao, T. and Fukui, S. An Outbreak of Epidemic Keratoconjunctivitis Due to Adenovirus Type $S$ in a Babies Home. Tohoku J. exp. Ned., 119 (2), 159 163 - Between October and December, 1973, an outbreak of epidemic keratoconjunctivitis oceurred in a babies home in Sapporo. 28 out of 58 infants and children suffered from the disease. Infection seemed to have been transmitted within a room by hands of nursing staff with spread into other rooms through the dispensary. Clinical manifestation in almost all of the patients was pseudomembraneous conjunctivitis without apparent corneal opacities. None of them showed systemic symptoms. Virus isolation was attempted from eye swabs of 4 patients between 5 and 8 days after onset of the disease, and adenovirus type 8 was isolated from all of the specimens. It was found that there was no case of subclinical infection by the serologic tests. Consecutive serologic studies revealed a good neutralizing antibody response to adenovirus type 8 in the patients tested. _- epidemic keratoconjunctivitis; type 8 adenovirus infection

Although epidemic keratoconjunctivitis (EKC) is not a rare disease in Japan, an outbreak in a group of infants has not been so far reported. This paper described epidemiological, clinical and laboratory studies on an outbreak of EKC in an infant community.

\section{Materials and Methods}

Subjects. There were 58 infants and children residing in The Hokkaido Central Babies Home in Sapporo City during the outbreak of EKC. They were divided in 4 rooms $(A, B, C$, and $D)$ according to their ages. Serum samples were collected from 48 infants at the early and late stages of the outbreak, and stored frozen at $-20^{\circ} \mathrm{C}$. Some of the patients were repeatedly bled over a period of 6 months after onset of the disease. Eye swabs were collected from 4 patients between 4 and 8 days after onset of the illness and stored frozen at $-20^{\circ} \mathrm{C}$ until used for virus isolation.

Virus isolation. Tubes of primary human embryonic kidney cell cultures were inoculated with the specimens and examined microscopically for cytopathic effect. Isolates thus obtained were then identified by the standard neutralization tests (Rose 1964) with rabbit antiserum to adenovirus type 8 .

Serologic tests. One of the isolated strains of adenovirus type 8 (strain U-T) was used for neutralization tests. HeLa cell cultures were employed for the tests and for

Received for publication, December $20,1974$. 
propagation of the virus. Neutralization tests were performed according to the standard method based on inhibition of cytopathic effect (Rose 1964). Complement-fixing antibodies were determined by the standard procedure (Casey 1965) using commercially obtained antigen (Type 5, Toshiba Chemical Co., Ltd.).

\section{Epidemiologtcal and Clinical Studies}

The first patient occurred in Room A on October 20, 1973. As shown in Fig. 1, infection was rapidly transmitted one after another until November 9. Only 2 infants in this room escaped the disease. The outbreak extended to Room $\mathrm{D}$ on November 15, and then to Room B on November 18. Since the first patient in Room $\mathrm{D}$ had been treated for style in the medical dispensary, infectious agent was supposed to be transmitted by hands of a doctor or a nurse. None of the infants and children in Room $D$ escaped the disease. Infection was transmitted to Room $B$ by an infant who was transferred from Room A during incubation period. Only 2 infants in this room, however, suffered from the disease.

The outbreak ceased at the early December. Finally, 28 out of 58 infants and children suffered from the disease. The youngest patient was a 3 month-old male. During the outbreak 3 nursing staffs ( 1 in Room B and 2 in Room D) were infected.

Clinical manifestation in almost all of the patients was pseudomembraneous conjunctivitis without apparent opacities. Cardinal symptoms were conjunctival ingestion, lidedema and flow of tears. Although corneal involvement was not apparent, some patients became photophobic. Symptoms were confined to unilateral eye in a small number of cases. None of the patients showed fever, cough or diarrhea.

Room A

(12)

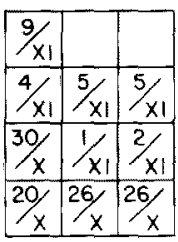

$2 \sim 10$ mo. old
Room B

(15)

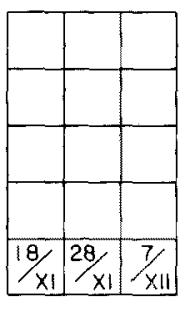

$6 \sim 12$ mo. old
Room C

$(16)$

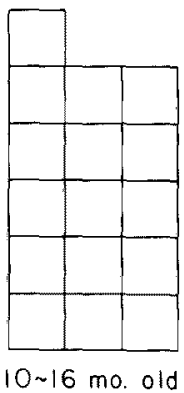

Room D

(15)

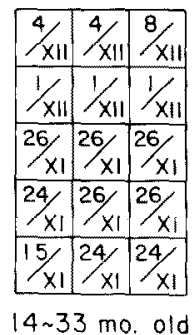

Fig. 1. Outbreak of epidemic keratoconjunctivitis in the Babies Home.

( ). number of infants; $\square$. without date, infant escaped from $\mathrm{EKC}$; $\square$ with date, patient. The dates of onset of the disease are indicated in the patients blocks.

\section{LABORATORY RESULTS}

Adenovirus type 8 was isolated from all of the specimens of 4 patients. Serum specimens collected at the end of outbreak were tested for neutralizing 

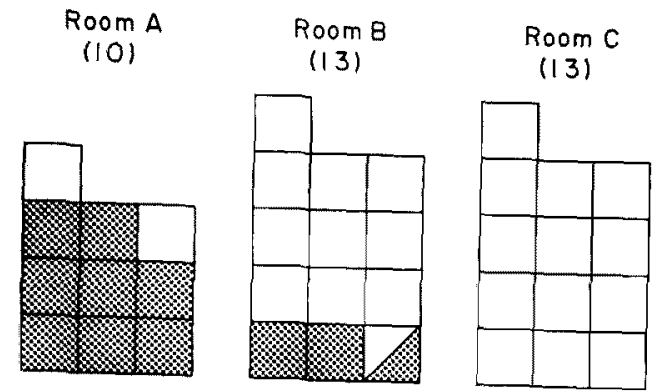

Room D

(I2)

Fig. 2. Results of the tests for neutralizing antibody to adenovirus type 8 in the sera collected at the end of the outbreak.

( ), number of tested subjects;

, patient, positive at $1: 4$ serum dilution:

patient, negative for antibody; $\square$, infant escaped from $\mathrm{EKC}$, negative for antibody.

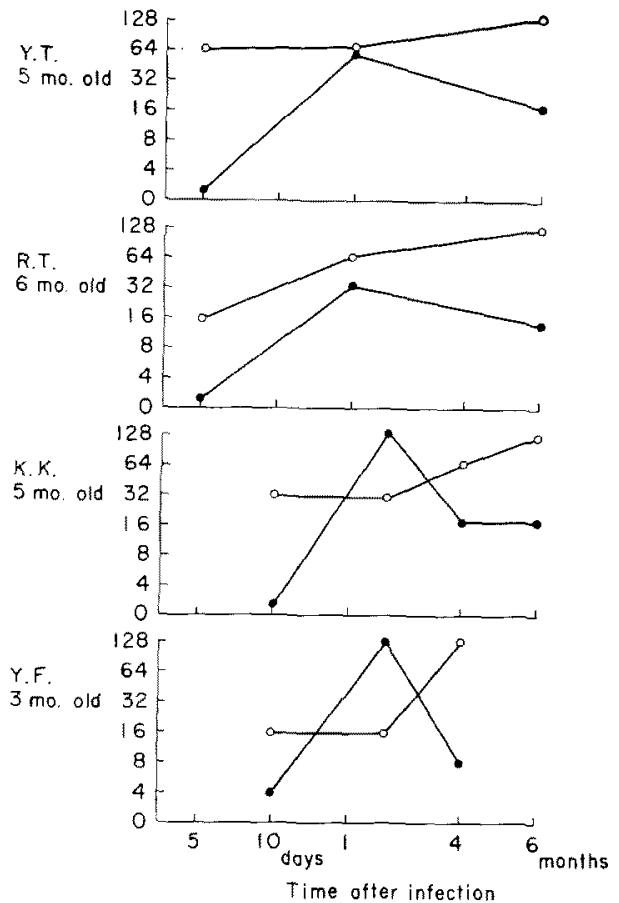

Fig. 3. Development of neutralizing and complement-fixing antibodies in 4 infants with epidemic keratoconjunctivitis. $0-0$, complement-fixing antibody; $\bullet \bullet$, neutralizing antibody.

antibody against adenovirus type 8 at 1:4 serum dilution. As shown in Fig. 2, all the infants in Room $A$ who became ill had neutralizing antibodies. In Rooms $B$ and $D$, neutralizing antibodies were not demonstrated in the sera which were obtained from the patients during incubation period or within several days after 
clinical onset. The same serum specimens were tested for complement-fixing antibodies to the adenovirus group common antigen. In Room $A$, the results coincided with those of neutralizing tests. In the other rooms, however, many infants and children who escaped the disease had complement-fixing antibodies.

Fig. 3 shows developments of neutralizing antibodies in 4 cases which could be followed up for relatively long period. Neutralizing antibodies were not detected within 10 days after clinical onset, then increased and reached to maximal titers ranging from $1: 32$ to $1: 128$ approximately one month later, and gradually decreased thereafter. Complement-fixing antibodies were already demonstrated in the acute stage of illness, and then gradually increased through the period of 6 months.

\section{Discussion}

It is well known that epidemic keratoconjunctivitis caused by type 8 adenovirus is mainly transmitted in an eye clinic by hands of physicians or nurses (Dawson et al. 1970). Sprague et al. (1973) recently reported that the bottle of ophthalmic wash solution contaminated with the virus was the major vehicle in the industrial outbreak in Alabama. Infection in the outbreak reported above also seems to have been transmitted within a room by hands of nursing staff with spread into other rooms through the dispensary. Thus, the mode of spread in this outbreak was different from that of other infections in this home such as mumps and varicella (Ikeda et al. 1971, 1973). An adequate hand washing of nursing staff before and after their handling each infant seemed to be the most effective measure for prevention of the spread of $\mathrm{EKC}$. Additional care for protection of infants from their direct contacts may be helpful to minimize the spread of infection in such a close community. Epidemiological studies in this outbreak support that such preventive measures must have been more strictly carried out in Room B which the source of infection was brought into later. Clinical manifestation of EKC in children is frequently a pseudomembraneous conjunctivitis without opacities but with systemic symptoms such as fever, cough or diarrhea (Mitsui et al. 1959). In this outbreak, however, we could observe neither apparent corneal opacities nor systemic symptoms in the patients.

Type 8 adenovirus can grow in various cell lines, but human embryonic kidney is said to be the most sensitive tissue for isolation of the virus (Sprague et al. 1973). This is also supported by the satisfactory results of virus isolation in this study.

Serologic tests revealed that all the infants were susceptible to type 8 advenovirus infection, and that there was no case of subclinical infection. Schmidt et al. (1966) reported that homotypic neutralizing antibodies to certain adenovirus types were not demonstrable in a higher proportion of patients in the group of infants and young children than in the older age group. This study, however, revealed good neutralizing antibody response to adenovirus type 8 even in infants. In contrast, the complement-fixation test was of little diagnostic value. 


\section{References}

1) Casey, H.L. (1965) Standardized diagnostic complement fixation method and adaptation to microtest. Public Health Monogr., 74, 1-34.

2) Dawson, C.R., Hanna, L., Wood, T.R. \& Despain, R. (1970) Adenovirus type 8 keratoconjunctivitis in the United States. Amer. J. Ophthal., 69, 473-480.

3) Ikeda, S., Chiba, S., Chiba, Y.\& Nakao, T. (1971) Epidemiological clinical and serological studies on epidemic of mumps in an infant nursery. Tohoku J. exp. Med., 105, 327-337.

4) Ikeda, S., Agastuma, Y., Wataya, Y. \& Nakao, T. (1973) Serological studies on two epidemics of varicella in a babies home. Tohoku J. exp. Med., 111, 79-85.

5) Mitsui, Y., Hanna, L., Hanabusa, J., Minoda, R., Ogata, S., Kurihara, H., Okamura, R. \& Miura, M. (1959) Association of adenovirus type 8 with epidemic keratoconjunctivitis. Special reference to the infantile form of the disease. Arch. Ophthal., 61, 891-898.

6) Rose, H.M. (1964) Diagnostic Procedures for Viral and Rickettsial Diseases. 3rd ed., American Public Health Association, New York, pp. 434-469.

7) Schmidt, N.J., Lennette, E.H. \& King, C.J. (1966) Neutralizing, hemagglutinatinginhibiting and group complement-fixing antibody responses in human adenovirus infections. $J$. Immunol., 97, 64-74.

8) Sprague, J.B., Hierholzer, J.C., Currier, R.W. II, Hattwick, M.A.W. \& Smith, M.D. (1973) Epidemic keratoconjunctivitis. A severe industrial outbreak due to adenovirus type 8. New Engl. J. Med., 289, 1341-1346. 